\title{
Análisis Factorial Exploratorio de la Escala Para Sucesos Traumáticos Colectivos (TEPT), Para Juventudes en Escenarios Violentos
}

\author{
Leticia Ríos-Velasco Moreno (PhD) \\ Universidad Autónoma de Ciudad Juárez, Instituto de Ciencias Sociales y \\ Administración \\ Departamento de Ciencias Sociales Av. Universidad y Heroico Colegio \\ Militar, Col. Foviste Chamizal, Cd. Juárez, Chih., México \\ Sarah Margarita Chávez-Valdez (PhD)
}

Escuela Libre de Psicología, A.C., Chihuahua ELPAC (Escuela Libre de

Psicología, A.C.) Universidad de Ciencias del Comportamiento,

Departamento de Comunicación Social, Calle Camino a Universidad La

Salle Núm., Col. Labor de Terrazas, Chihuahua, Chih., México

Oscar Armando Esparza-del Villar (PhD)

Beatriz Maldonado-Santos (PhD)

Lucia Nereth Quintana-Moye (PhD)

Universidad Autónoma de Ciudad Juárez, Instituto de Ciencias Sociales y Administración

Departamento de Ciencias Sociales Av. Universidad y Heroico Colegio

Militar, Col. Foviste Chamizal, Cd. Juárez, Chih., México

Doi:10.19044/esj.2019.v15n16p217 URL:http://dx.doi.org/10.19044/esj.2019.v15n16p217

\section{Resumen}

Una amplia serie de síntomas en grupos de jóvenes, que pueden ser etiquetados como "rasgos colectivos", sugieren la necesidad de explorar y validar un instrumento de evaluación para jóvenes en vulnerabilidad social, con un alto nivel de ansiedad social y estrés postraumático ante la exposición directa e indirecta al delito. En un contexto de alto impacto, y afrontamiento físico-afectivo asociado al trastorno de estrés postraumático, las reacciones prototípicas del trastorno al igual que particularidades sui generis de carácter contextual configuraron dichos rasgos en nuestra muestra. La validación se realizó en las ciudades de Chihuahua y Cd Juárez, Chihuahua, a una muestra de 396 jóvenes, 178 hombres y 218 mujeres, con edad media de 19.5 $(\mathrm{DE}=1.24)$, pertenecientes a instituciones universitarias públicas y privadas, de las cuales $54.2 \%$ eran públicas. Gracias al muestreo por conglomerados y la selección aleatoria, las mediciones se recabaron durante un periodo de 
cuatro meses. El 37.25\% del total de la varianza, corresponde a síntomas de: angustia-desesperanza. Un segundo factor explica el $10.42 \%$ del total de la varianza referente a sintomatología de reexperimentación-somatización. Un $5.59 \%$ del total de varianza hace referencia a síntomas de aislamiento social, y el cuarto factor explica el $5.30 \%$ de varianza total, que constata evitación en la muestra. Finalmente, un quinto factor explica el $4.44 \%$ del total de la varianza, referente a la hipervigilancia. La escala de estrés postraumático colectivo para eventos traumáticos en su análisis factorial exploratorio indica índices de confiabilidad que la convierten en un instrumento confiable en la medición de rasgos traumáticos colectivos para jóvenes en situación de violencia.

Palabras clave: Validación; Trauma colectivo; Juventudes; Victimización

\section{Exploratory Factor Analysis (EFA) Youth Posttraumatic Scale for Collective Trauma in Violent Scenarios}

\section{Leticia Ríos-Velasco Moreno (PhD)}

Universidad Autónoma de Ciudad Juárez, Instituto de Ciencias Sociales y Administración

Departamento de Ciencias Sociales Av. Universidad y Heroico Colegio

Militar, Col. Foviste Chamizal, Cd. Juárez, Chih., México

\section{Sarah Margarita Chávez-Valdez (PhD)}

Escuela Libre de Psicología, A.C., Chihuahua ELPAC (Escuela Libre de

Psicología, A.C.) Universidad de Ciencias del Comportamiento,

Departamento de Comunicación Social, Calle Camino a Universidad La

Salle Núm., Col. Labor de Terrazas, Chihuahua, Chih., México

Oscar Armando Esparza-del Villar (PhD)

Beatriz Maldonado-Santos (PhD)

Lucia Nereth Quintana-Moye (PhD)

Universidad Autónoma de Ciudad Juárez, Instituto de Ciencias Sociales y

Administración

Departamento de Ciencias Sociales Av. Universidad y Heroico Colegio

Militar, Col. Foviste Chamizal, Cd. Juárez, Chih., México 
Abstract

A wide range of symptoms found in youth groups, which can be labeled as "collective traits", suggest the need to explore and validate an assessment tool for these groups and those in social vulnerability. This is with high levels of social anxiety and posttraumatic stress in the face of direct and indirect exposure to crime. In the context of high-impact crimes and physioemotional coping associated with PTSD, the prototypical reactions of the disorder, coupled with sui generis contextual characteristic traits, configured our exploratory sample. Validation of the instrument was conducted in the cities of Chihuahua and Ciudad Juarez, Mexico, on a sample of 396 young people (178 men and 218 women with a mean age of $19.5(\mathrm{SD}=1.24)$ ), belonging to public and private academic institutions, in which $54.2 \%$ belonged to public institutions. Cluster sampling, random selection, and measurements were collected during a period of four months. The $37.25 \%$ of the total variance corresponds to symptoms of anguish-despair. A second factor explained $10.42 \%$ of the total variance with five items with symptomatology of reexperimentation-somatization. A $5.59 \%$ of the total variance also includes symptoms of social isolation. The fourth factor explained $5.30 \%$ of variance, with items about avoidance. In addition, a fifth factor explained $4.44 \%$ of the total variance, referring to hypervigilance. The collective post-traumatic stress scale for traumatic events in this exploratory factor analysis indicated reliability indices that guarantee a reliable instrument in the measurement of collective traumatic traits for youths in situations of violence.

Keywords: Validation, Collective trauma, Youths, Victimization

\section{Introducción}

La ola de violencia registrada en el año 2017 en México se prolongó hasta el año 2018 registrando 33,341 asesinatos, siendo este el año más violento de la historia de México desde 1997, año en el que empezaron los registros históricos. Las cifras promedio de 80 asesinatos diarios en el año 2017 se incrementaron a 92 asesinatos promedio al día para el año 2018, cifras que fueron publicadas recientemente por el Secretariado Ejecutivo del Sistema Nacional de Seguridad Pública, 2019.

En los estados del Norte de México,especialmente en el estado de Chihuahua, la violencia se ha recrudecido y es una constante, tal es el caso de las ciudades de: Chihuahua y Cd. Juárez, Chihuahua. En dicho contexto, la victimización directa e indirecta, en particular en su franja fronteriza, ha sido descrita por los investigadores Miranda, Moreno, Mera, Palacios y López 
(2010), comoun panorama de alta afectación contextual por la alta exposición a delitos de alto impacto por más de una década, detectándose en los ciudadanos ciertas creencias, actitudes y comportamientos, en especial en los jóvenes de 15 a 21 años, que parecen encajar con algunos rasgos de ansiedad, fobias, estrés agudo y depresión, todos diagnósticos de alto perfil clínico, tradicionalmente individuales, que sintomáticamente, y en suma, a nivel colectivo, denotan rasgos de afectación mental, esto con implicaciones directas en sus relaciones, identidad, bienestar y desarrollo pleno, victimizando familias y comunidades enteras para las regiones norteñas de México, especialmente aquellas del estado de Chihuahua ( Miranda et al., 2010).

En torno a la psicobiología de la violencia se ha encontrado que, en contextos de violencia, los ciudadanos se ven sometidos a niveles altos de estrés, produciéndose efectos en la señalización de ciertos neurotransmisores como: la adrenalina, la noradrenalina y el cortisol, los cuales tienen que ver con la respuesta del cerebro ante situaciones de peligro y que fungen como factores de supervivencia durante aquellas circunstancias en que se produce una agresión. De igual manera, se ha descubierto que, a largo plazo, dichos sujetos sometidos a prolongada exposición de episodios violentos, sufren un descontrol en la producción de neurotransmisores, derivando en desequilibrios letales (Huntington, 2012).

Estudios previos en la zona norte de México, específicamente en las ciudades de Chihuahua y Cd. Juárez, realizados por Chávez y Ríos Velasco (2015), detectaron en la población "conductas que atentan al equilibrio mental de sus pobladores, como es el caso de emociones y comportamientos contrarios a una adaptación positiva a la adversidad como: evitación, rumiación, pasividad, frustración, miedo-ansiedad, ira, depresión-tristeza" (p.30).

Según Piqueras-Rodríguez, Ramos-Linares, Martínez- González y Oblitas (2009) estas reacciones tienen una función preparatoria para dar una respuesta adecuada a las demandas del ambiente; son respuestas eminentemente adaptativas para el individuo. Sin embargo, en ocasiones encontramos que algunas de ellas pueden transformarse en patológicas para ciertos individuos, debido a un desajuste en la frecuencia o intensidad en un intento de adecuarse al contexto adverso. Cuando tal desajuste acontece y se mantiene, puede sobrevenir un trastorno de la salud tanto mental, como es el caso de: los trastornos de ansiedad, depresión mayor, ira patológica, etcétera, como físicos; tal el caso de: trastornos cardiovasculares, reumatológicos, inmunológicos, etcétera (Piqueras-Rodríguez et al., 2009).

Para Bonilla y Fernández-Guinea (2006), los trastornos biológicos de la inmunodeficiencia adquirida por el sistema autoinmune, la esclerosis múltiple, la diabetes juvenil y un centenar de trastornos, proporcionan una 
metáfora de las alteraciones físicas y disfunciones en el sistema relacionadas con las defensas emocionales, cognitivas y conductuales de un joven. La adaptación ahora está orientada a la satisfacción de la necesidad, de manera indirecta, pero en forma directa se enfrenta la adversidad.

Para Chávez y Ríos Velasco (2015), la ilegalidad, intolerancia, falta de respeto por las normas sociales de convivencia, veneración a ideologías y entornos nocivos, han afectado en suma a nivel comunitario, al igual que la exposición, directa o indirecta, a una serie de eventos con alto contenido traumático; tal es el caso de delitos de alto perfil, como: desapariciones, secuestros, homicidios, agresiones sexuales, robos a mano armada, extorsión, entre otros, generalizándose así los estados de estrés e incorporándose de manera estructural en las comunidades una alta y crónica vulnerabilidad social.

Pascual, Conejero y Etxebarria (2016), defienden que la adecuación de una determinada estrategia de afrontamiento o de regulación emocional debe hacerse teniendo en cuenta el contexto en que se produce y en qué medida la persona logra disminuir la ansiedad, quedar satisfecha consigo misma y mejorar sus relaciones sociales, encontrando que las estrategias pasivas, es decir, el hecho de centrarse en las propias emociones (evitación, negación, ilusión, rumiación, inacción y escape) se asocian de forma significativa y negativa hacia el logro de un afrontamiento adaptativo (Pascual et al., 2016). Esto representa una amenaza en el cúmulo de emociones físicas, cognitivas y conductuales, con implicaciones en el bienestar y la calidad de vida, acentuándose y acrecentándose dichos niveles de estrés postraumático en los más vulnerables.

Para Páez, Vázquez y Echeburúa (2013) es posible lograr crecimiento postraumático, especialmente en colectivos, bajo las siguientes premisas: que el trauma sea de carácter social y la cultura de corte colectivista, especialmente en personas que aún estén construyendo su identidad, tengan recursos ideológicos, y no sean comunidades desorganizadas; que la severidad del estrés sea media, no extrema, donde haya apoyo y participación social, y una comunicación proactiva entre los miembros que apoye a una construcción narrativa de la problemática (Páez et al., 2013). Sin embargo, los niveles de estrés y adaptación al entorno violento no son los adecuados, el rango etario y vulnerabilidad del contexto no permiten controlar los niveles de trauma por estrés en dichas zonas afectadas por una violencia extrema y se ha disminuido la unión y fuerza para reconstuir el tejido social en las zonas mencionadas.

En este sentido, el presente estudio se enfoca en validar, mediante un análisis factorial exploratorio, la escala para sucesos traumáticos colectivos (TEPT), una escala dirigida a colectivos expuestos a violencia contextual. Con este estudio se pretende obtener un instrumento operativo, breve y de fácil aplicación, que permita medir en juventudes mexicanas los factores del 
constructo denominado estrés postraumático, con sus particularidades contextuales, con el fin de ser una herramienta dirigida, útil y confiable para medir dichos rasgos en los jóvenes en el territorio de México, especialmente en las zonas fronterizas con el norte de México.

\section{Método}

Para el diseño se partió particularmente de un enfoque cuantitativo. El tipo de diseño del presente estudio es de análisis de propiedades psicométricas de un instrumento, no experimental, de campo. Se realizó un análisis factorial exploratorio (AFE) mediante el método de mínimos cuadrados generalizados con tipo de rotación oblicua tipo Promax; así mismo, se efectuó la prueba de esfericidad de tipo Kaiser-Meyer-Olkin.

\section{Participantes}

Se realizó el estudio con 396 jóvenes de 17 a 25 años de edad, con una media de edad de 19.5, de los cuales 178 fueron hombres y 218 mujeres, algunos estudiantes de la Universidad Autónoma de Juárez, la Universidad Autónoma de Chihuahua y la Universidad Regional del Norte, de las ciudades de Chihuahua y Cd. Juárez, Chihuahua. En específico, de la muestra final de 396 jóvenes, 102 jóvenes reportaron estar laborando mientras que 294 jóvenes no laboran; de los que laboran el $42.23 \%$ genera menos de $\$ 3000$ pesos al mes (65 jóvenes), mientras que el $35.54 \%$ genera de $\$ 3500$ a $\$ 5000$ (100 jóvenes), el $22.23 \%$ de los jóvenes gana entre $\$ 5000$ y $\$ 10,000$ pesos mexicanos, de los cuales $54.2 \%$ provenían de escuelas públicas, y un $45.8 \%$ de escuelas privadas. El muestreo se llevó a cabo por conglomerados, selección aleatoria; las mediciones se recabaron durante un periodo de cuatro meses.

\section{Instrumento}

La escala para sucesos traumáticos colectivos (TEPT)fue desarrollada por Pineda, Guerrero, Pinilla y Estupiñán (2002); se trata de una escala breve que consta de 24 ítems, los cuales se responden mediante un proceso de auto informe que, en suma, se ajustan a características que se basan en el diagnóstico del estrés postraumático en poblaciones violentas. Esta escala ha sido utilizada para población juvenil y adulta que habita áreas conurbadas y ejidales sometidas a violencia contextual. La escala de respuesta que maneja es tipo Likert.

La escala TEPT para eventos colectivos traumáticos tiene una buena capacidad de discriminación y buenos niveles de sensibilidad, especificidad y ajuste. La lista de síntomas presenta una consistencia interna aceptable, en el estudio de Chávez y Ríos Velasco (2015), se reporta una alfa de Cronbach de 0.92. Esta escala no ha sido anteriormente validada en población mexicana. 


\section{Procedimiento}

Los centros fueron contactados dos meses antes del proceso de recolección de datos, esto después de obtener el permiso de la Universidad Autónoma de Juárez, la Universidad Regional del Norte y la Universidad Autónoma de Chihuahua y solicitando el consentimiento informado de los jóvenes, por medio de una carta y entrevista previa.

La escala para sucesos traumáticos colectivos (TEPT) fue completada por los jóvenes en un período de 30 a 50 minutos. Después de recolectar la información se generó la base de datos que fue analizada por medio del paquete estadístico SPSS versión 23. Se procedió luego a realizar un análisis factorial exploratorio que demostró un buen ajuste del modelo, las cargas factoriales y la validez del coeficiente de Cronbach de cada uno de los elementos que conforman la escala.

\section{Resultados}

Se realizó un Análisis Factorial Exploratorio (AFE) forzando la estructura a cuatro y a cinco factores. Se utilizó el método de mínimos cuadrados generalizados con rotación oblicua tipo Promax (MacCallum, Widaman, Zhang \& Hong, 1999). Al analizar las dos estructuras factoriales se decidió que la solución de cinco factores presentaba una mejor organización de los ítems (Tabla 1).

Tabla 1. Análisis Factorial Exploratorio del Cuestionario TEPT

\begin{tabular}{|c|c|c|c|c|c|}
\hline \multirow[b]{2}{*}{ Reactivos } & \multicolumn{5}{|c|}{ Factor } \\
\hline & 1 & 2 & 3 & 4 & 5 \\
\hline 11a. A partir de lo que pasó, siento que nada me importa & $\mathbf{0 . 7 3}$ & 0.40 & 0.51 & 0.25 & 0.31 \\
\hline $\begin{array}{l}\text { 11b. Después de la situación, tengo muchas dificultades para } \\
\text { llevar a cabo las actividades que hacía antes }\end{array}$ & 0.74 & 0.43 & 0.55 & 0.23 & 0.45 \\
\hline $\begin{array}{l}\text { 12. A partir de lo que pasó, las personas que me rodean ya no } \\
\text { son importantes para mí }\end{array}$ & $\mathbf{0 . 8 1}$ & 0.33 & 0.52 & 0.14 & 0.31 \\
\hline $\begin{array}{l}\text { 13. Después del suceso desagradable tengo muchas dificultades } \\
\text { para querer como lo hacía antes }\end{array}$ & 0.78 & 0.43 & 0.58 & 0.24 & 0.38 \\
\hline $\begin{array}{l}\text { 14. A partir de lo que sucedió, siento que mi futuro es triste y } \\
\text { desolador }\end{array}$ & 0.78 & 0.38 & 0.52 & 0.07 & 0.37 \\
\hline $\begin{array}{l}\text { 15. Después del suceso desagradable, me es muy difícil } \\
\text { conciliar el sueño }\end{array}$ & 0.73 & 0.50 & 0.55 & 0.11 & 0.41 \\
\hline $\begin{array}{l}\text { 16. Después de la situación que viví, siento que frecuentemente } \\
\text { estoy de mal humor }\end{array}$ & 0.75 & 0.47 & 0.63 & 0.10 & 0.50 \\
\hline $\begin{array}{l}\text { 17. Me es muy difícil concentrarme en mis actividades, } \\
\text { después de lo sucedido }\end{array}$ & 0.72 & 0.49 & 0.62 & 0.14 & 0.55 \\
\hline $\begin{array}{l}\text { 3. Constantemente tengo pensamientos que me recuerdan la } \\
\text { situación desagradable y me provocan mucha angustia }\end{array}$ & 0.24 & 0.65 & 0.25 & 0.55 & 0.37 \\
\hline 4. Sueño mucho con lo que pasó & 0.43 & 0.85 & 0.38 & 0.34 & 0.41 \\
\hline 5. La mayor parte del tiempo creo estar viviendo lo sucedido & 0.53 & 0.83 & 0.47 & 0.26 & 0.35 \\
\hline 6. Cuando algo me recuerda la situación, me siento muy mal & 0.21 & 0.63 & 0.26 & 0.52 & 0.47 \\
\hline
\end{tabular}


7. Cuando algo me recuerda un aspecto de la situación, mi cuerpo se altera

20a. Después de esa situación, la mayor parte del tiempo me siento mal, en todos los sentidos

20b. He disminuido casi todas mis actividades sociales después de lo que me pasó

20c. Después de lo sucedido, tengo muchas dificultades en mis relaciones con los demás

20d.Después de lo que me pasó, he disminuido en gran medida mi ritmo de trabajo

1. Últimamente he vivido al menos una situación relacionada con muertes o amenazas contra mi vida o la de otras personas relacionadas conmigo

2. Por esta situación he experimentado mucha angustia o temor excesivo

8. Siempre evito pensar o hablar de lo que pasó

9. La mayoría de las veces evito cosas y sitios que me recuerden la situación

18. Desde que tuve esa situación horrible, siempre observo con sospecha todo lo que ocurre a mi alrededor

19. Desde aquél acontecimiento, cualquier cosa me pone en alerta y me asusta

Reactivo Descartado

10. Olvidé muchas cosas de la situación desagradable

\begin{tabular}{|l|l|l|l|l|}
0.39 & $\mathbf{0 . 6 6}$ & 0.43 & 0.40 & 0.48 \\
0.56 & 0.47 & $\mathbf{0 . 8 2}$ & 0.11 & 0.49 \\
0.57 & 0.37 & $\mathbf{0 . 8 0}$ & 0.27 & 0.46 \\
0.60 & 0.40 & $\mathbf{0 . 8 2}$ & 0.16 & 0.47 \\
0.64 & 0.36 & $\mathbf{0 . 8 3}$ & 0.21 & 0.36 \\
0.18 & 0.32 & 0.20 & $\mathbf{0 . 4 4}$ & 0.23 \\
0.18 & 0.47 & 0.32 & $\mathbf{0 . 6 4}$ & 0.31 \\
0.12 & 0.25 & 0.15 & $\mathbf{0 . 5 4}$ & 0.22 \\
0.16 & 0.25 & 0.14 & $\mathbf{0 . 5 1}$ & 0.26 \\
0.41 & 0.36 & 0.44 & 0.28 & $\mathbf{0 . 7 7}$ \\
0.39 & 0.48 & 0.47 & 0.31 & $\mathbf{0 . 8 5}$ \\
0.15 & 0.12 & 0.08 & 0.23 & 0.01 \\
\hline
\end{tabular}

Nota: Las cargas factoriales más altas aparecen en Negritas

En este modelo de cinco factores, los índices de Kaiser-Meyer-Olkin $(\mathrm{KMO}=0.92)$ y de la prueba Bartlett $(\mathrm{p}<0.01)$ nos indican que el modelo es apropiado y no presenta esfericidad. Se analizaron los pesos factoriales de los reactivos en cada uno de los cinco factores y de los 24 sólo se eliminó un reactivo. El reactivo 10 se eliminó ya que el peso factorial más alto fue de 0.23 el cual es un valor bajo. El rango de los pesos factoriales del resto de los reactivos fue de 0.44 a 0.85 . El análisis factorial exploratorio muestra que los ítems convergen y discriminan entre sí y nos muestran cinco factores/ constructos a considerar para la Escala de sucesos traumáticos colectivos (TEPT), para juventudes en escenarios violentos. (angustia-desesperanza, re experimentación-somatización, aislamiento social, evitación e hipervigilancia).

\section{Confiabilidad}

Se calculó la confiabilidad interna de cada uno de los factores con el método de obtención de alfa de Cronbach. El factor 1 tuvo una consistencia interna de $\alpha=0.91$; el factor 2, $\alpha=0.84$; el factor 3, $\alpha=0.88$; el factor $4, \alpha=$ 0.60 ; el factor $5, \alpha=0.79$. La confiabilidad interna del total de los reactivos fue $\alpha=0.85$; la mayoría de los valores presentan una consistencia interna aceptable a excepción del factor 4. 


\section{Conclusiones y Discusión}

Se confirmó que la estructura de los rasgos de estrés postraumático en colectivos vulnerados por violencia social puede representarse por un modelo jerárquico en el que cinco factores de primer orden configurarían su estructura: un primer factor que explica el $37.25 \%$ de la varianza, cuyos pesos factoriales van de 0.72 a 0.81 , compuesto por ocho reactivos, refirió sentimientos de angustia-desesperanza y efectos físico-emocionales en el colectivo, siendo este primer factor el que principalmente representó los rasgos más importantes, basándonos en los pesos factoriales y porcentaje de varianza. El segundo factor, compuesto por aquellos rasgos de reexperimentaciónsomatización y alteraciones asociadas propias al trastorno, a nivel colectivo encuentra una explicación del $10.42 \%$ del total de la varianza con pesos factoriales que oscilaron de 0.63 a 0.85 . El tercer factor explica el $5.59 \%$ del total de la varianza, con rango de pesos factoriales que van de 0.80 a 0.83 , refiere aislamiento social, considerando a su vez, las implicaciones derivadas del malestar personal y social al que se encuentran sometidos los colectivos vulnerables. El cuarto factor referente a reactivos de evitación, explica un $5.30 \%$ del total de la varianza con pesos factoriales entre 0.44 a 0.64 y el quinto factor con el $4.44 \%$ del total de varianza explicada con pesos factoriales de 0.77 a 0.85 , se refiere a reactivos de hipervigilancia que miden el estado de alarma y amenaza percibida constante en dichos colectivos.

Respecto al clima de violencia contextual imperante en las comunidades de Chihuahua, México donde se realizó este estudio, y en referencia a una posible resiliencia y crecimiento social ante el trauma, señalados por Páez et al. (2012), se establecen algunos aspectos que podrían detonar un crecimiento postraumático en colectivos enfrentados a violencia contextual, como sería que el acontecimiento sea colectivo y suscitado en sociedades colectivistas. En este sentido, se identifica que aunque el trauma sea de carácter social, en comunidades colectivistas, y en muestras de jóvenes, aún en la construcción de su identidad, no se identifican recursos ideológicos que apunten a un afrontamiento proactivo y de renovación comunal del tejido social, puesto que se trata de comunidades con perfil y rumbo difuso en su organización social donde se carece del apoyo, participación, y con niveles bajos de cohesión social (unión y fuerza). A esto se une la infiltración de la delincuencia en sus vecindarios, la cual resulta un agravante adicional, así como los niveles nulos o muy bajos de interés por la intervención comunitaria, elementos básicos para una adecuada eficacia colectiva, y la ambigüedad diaria en su contexto, dificultando la renovación en dichos colectivos mediante una narrativa positiva de la problemática.

Este estudio analiza la estructura factorial y confiabilidad interna de un instrumento creado en Colombia con una situación social parecida, en la que 
se miden rasgos de estrés postraumático (TEPT) aplicados a la violencia social. En la literatura científica este fue el único instrumento validado en alguna parte de Latinoamérica que evaluaba TEPT en ambientes de violencia social. La importancia de validar este instrumento en ciudades como Chihuahua y Juárez, México, zonas de alta violencia contextual, permite corroborar su validez para poder ser utilizado en el análisis de aspectos psicológicos del trastorno en los Chihuahuenses y Juarenses, y abre la posibilidad a estudios posteriores y respectivos análisis exploratorios, con sus respectivas adecuaciones de léxico y contextualización, para muestras de otros rangos etarios y de zonas similares con afectaciones ante la crisis de violencia imperante en México.

\section{Limitaciones y Sugerencias}

Una limitación del estudio es que se realizó solamente con una población estudiantil universitaria. Esto limitaría la generalización a personas entre los 17 y 25 años aproximadamente, en su mayoría solteras, sin hijos, y a personas que tengan un nivel educativo universitario. Es importante tomar muestras diferentes a la universitaria que incluyan a gente más joven, y más grande, que cuenten con diversos niveles educativos, otros estados civiles, y que sean además jefes de familia. Así como Chihuahua y Juárez presentan violencia social, existen otras ciudades de México y Latinoamérica con realidades similares en las cuales se tendría que analizar la confiabilidad y validez del instrumento. Estas preguntas empíricas se tienen que responder validando el instrumento con otros tipos de poblaciones.

También es importante aplicar el instrumento en una muestra distinta de las mismas ciudades para confirmar la estructura factorial a través de un análisis factorial confirmatorio y así corroborar la estructura en esta población. Aunado a esto, es importante validar la validez convergente y concurrente del instrumento para ver su relación con otros instrumentos que midan constructos similares o relacionados al TEPT para así fortalecer más la validez del instrumento. Esta escala pretende ser un instrumento de mucha utilidad para aquellos que estudian las secuelas psicológicas del trauma debido a la violencia social en las comunidades.

Los resultados obtenidos en esta investigación permiten aseverar los siguientes aspectos:

1) El bajo nivel de omisión y la capacidad discriminativa de los ítems y las confiabilidades obtenidas, nos indica que la escala para sucesos traumáticos colectivos (TEPT), para juventudes en escenarios violentos es un instrumento confiable para medir los rasgos de estrés postraumático en juventudes expuestas a violencia contextual.

2) Respecto a la estructura teórica resultante en el Análisis Factorial Exploratorio (AFE), esta resultó concordante con la descripción teórica que 
brinda el DSM-V del constructo denominado “estrés postraumático“; por tanto, se sugiere seguir desarrollando instrumentos de medición en base a los mismos. Asimismo, se sugiere validar la estructura teórica obtenida en la escala para sucesos traumáticos colectivos (TEPT) a través de jueces expertos y que ésta a su vez sea mantenida, ya que el análisis detallado de cómo se agruparon los ítems indica que es la mejor explicación teórica sobre el instrumento.

3) Es importante seguir estudiando el trastorno de estrés postraumático en escenarios violentos a fin de comprender su influencia y procesos de desgaste en las comunidades lesionadas por el trastorno, además de los factores asociados a la variación de las percepciones.

\section{References:}

1. Barraza, L. \& Almada, H. (2012). La realidad social y las violencias en Ciudad Juárez. Ciudad Juárez, México: Universidad Autónoma de Ciudad Juárez.

2. Bonilla, J. \& Fernández-Guinea, S. (2006). Neurobiología y neuropsicología de las conductas antisociales. Revista psicopatología clínica, legal y forense, 6, (1-3), 67-81.

3. Chávez, S. \& Ríos Velasco, L. (2015). Intervención para el Desarrollo Socioemocional, la conducta prosocial y la inhibición de la conducta violenta en jóvenes. Sesión de posters presentada en la lera Conferencia Regional Latinoamericana de Psicología. "Avances y Desafíos para la Psicología en América Latina", Memorias del Congreso del Colegio Oficial de Psicólogos de Colombia, 921-922.

4. Huntington, J. (2012). Impact of exposure to Violence on Urban Youth: A Biopsychosocial Perspective of Aggression. Seton Hall University Dissertation and Theses.

5. Instituto Nacional de Geografía y Estadística (2017). Encuesta Nacional de Victimización y Percepción sobre Seguridad Pública (ENVIPE). Archivo Electrónico, México, 2017. Recuperado de "URL"

6. MacCallum, R., Widaman, K., Zhang, S. \& Hong, S. (1999). Sample size in factor analysis. Psychological Methods, 4, 84-99.

7. Miranda, S., Moreno, J., Mera, A., Palacios, M. \& López, D. (2010). Caracterización de los delitos comunes y del crimen organizado en las ciudades de Chihuahua y Ciudad Juárez. Chihuahua, México: Ediciones Quibit Cluster.

8. Páez, D., Vázquez, C. \& Echeburúa, E. (2013). Trauma Social, Afrontamiento Comunitario y Crecimiento Postraumático Colectivo. En Carrasco, M.J. y Charro, B. (Eds.). Crisis, vulnerabilidad y 
superación (pp. 15-50). Reflexiones Comillas. Psicología, 2, Madrid: Ediciones Universidad Pontificia Comillas.

9. Pascual, A., Conejero, S. \& Etxebarria, I. (2016). Coping strategies and emotion regulation in adolescents: Adequacy and gender differences. Ansiedad y Estrés, 22(1), 1-4.

10. Pineda, D.A., Guerrero, O. L, Pinilla, M.L. \& Estupiñán, M. (2002). Utilidad de un cuestionario para rastreo de estrés postraumático en una población colombiana. Revista de Neurología, 34(10), 911-916.

11. Piqueras-Rodríguez, J., Ramos-Linares, V., Martínez González, A., \& Oblitas G, L. (2009). Emociones Negativas y su Impacto en la Salud Mental y Física. Suma Psicológica, 16 (2), 85-112.

12. Secretariado Ejecutivo del Sistema Nacional de Seguridad Pública (2019). Centro Nacional de Información. Víctimas de Delitos del Fuero Común 2018: Instrumento para el Registro, Clasificación y Reporte de Delitos y las Víctimas CNSP/38/15. Recuperado de http://secretariadoejecutivo.gob.mx/docs/pdfs/nuevametodologia/CNSP-Víctimas-2018_dic18.pdf. 